Proceedings of the Creative Construction Conference (2018)

Edited by: Miroslaw J. Skibniewski \& Miklos Hajdu

DOI 10.3311/CCC2018-011

Creative Construction Conference 2018, CCC 2018, 30 June - 3 July 2018, Ljubljana, Slovenia

\title{
Process information modelling (PIM) for public housing construction project in Hong Kong
}

\author{
Wen Pan ${ }^{\mathrm{a}, *}$, Bahriye Ilhan ${ }^{\mathrm{b}}$, Thomas Bock ${ }^{\mathrm{a}}$ \\ ${ }^{a}$ Technical University of Munich, Chair for Building Realisation and Robotics, Arcisstrasse 21, Munich 80333, Germany \\ ${ }^{b}$ Istanbul Technical University, Architecture Faculty, Taskisla Cd. 2, Istanbul 34367, Turkey
}

\begin{abstract}
Public building construction, which consists of many sub-tasks and numerous systematised working processes such as planning, mobilisation, scheduling, procurement and controlling, is complicated. If the project team is incapable of managing these processes seamlessly, it may result in severe project delay and cost overrun. This issue becomes even more apparent when utilising construction robotics, since precise process and scheduling information as well as feedback is required to ensure each task is completed correctly and on time. Support in addressing these complex management activities in particular for the robotic usage offers Process Information Modelling (PIM). In this paper the process-oriented modelling approach, PIM, which provides a collaborative way of planning, designing, producing, assembling and entire project life cycle management strategy is introduced. The main objective of PIM is to integrate with the conventional Building Information Models (BIM) and supplement them with a process oriented database platform, allowing for smooth data transfer, as well as promoting seamless and constant data sharing among all stakeholders. Digital documentation, simulation and real-time data are produced progressively to support the decisionmaking process. The effectiveness of the PIM is demonstrated on façade painting task by a painting robot for an on-going consultancy project commissioned by the construction industry council (CIC) in Hong Kong. The impacts of PIM on supporting the potential future applications of construction robotics and instigating the next construction information evolution are discussed.
\end{abstract}

(C) 2018 The Authors. Published by Diamond Congress Ltd., Budapest University of Technology and Economics Peer-review under responsibility of the scientific committee of the Creative Construction Conference 2018.

Keywords: construction automation; construction management; Hong Kong; integration; PIM

\section{Introduction}

For many years, many researchers have agreed that efficient integration and coordination of design, construction, and management data can potentially benefit the overall performance of the construction industry [1]. Recently, other industries are submerged with data; the construction industry is not an exception to this unprecedented trend. In each step of a construction project, the project team will be dealing with enormous data, which amongst various professions and the decisions have been made could impose serious implications on the construction project. Currently, Building Information Modelling (BIM) technologies are being considered capable to deal with multidimensional CAD information systematically, improve data integration among cross-disciplinary collaboration across the construction industry and between the key stakeholders. 
Even though BIM can cover the construction project span from early stages to the completion of the project, the implementation of BIM technologies is often fragmented in most of the construction projects. This is partly due to the fact that in practice BIM does not effectively categorise or integrate the most relevant data and distribute the information to the most desired stakeholder. A piece of information cannot function alone without specific protocols and relationships behind it [2]. The BIM technologies should be able to provide a platform where the data collected from each phase of the project that can be integrated as well as interoperating between various applications [3]. Therefore, the concept of the next generation of BIM is not merely just gathering information or use of several of technical tools but managing information across diverse collaboration and interrelationship of the key stakeholders. Additionally, it is essential to deliver the right information to the right place for the right people at the right time [4]. Process Information Modelling (PIM) has the potential to be developed as the next generation BIM, which will enhance information integration, yet focus on the process of each construction tasks and the relationship between each attribute. In this case, the attributes can be people, products, process or technologies. By doing so, it helps the project team to identify challenges of execution from both technological and social approach also provides feasible solutions proactively [5]. The detailed description about PIM is demonstrated in the later section.

This paper proposes a scenario in conjunction with the ongoing consultancy project commissioned by the Construction Industry Council (CIC) in Hong Kong. It hypothesises the PIM concept imperatively applied to the utilization of a façade painting robot. In general, the Hong Kong Public Housing Construction (PHC) sector faces experiences three major challenges: (1) to satisfy the increasing demand; (2) to achieve affordable price and (3) to address demographic changes. Accordingly, the CIC commissioned the Chair of Building Realization and Robotics $\left(\mathrm{br}^{2}\right)$ at Technical University of Munich (TUM) to research and develop construction robots and automation strategies that are tailor-made for the PHC in Hong Kong. The proposed external painting robot provides an opportunity to develop and validate the PIM concept. The implementation of construction robotics will trigger a series of changes in the construction sequence and potentially revolutionises the construction industry as a whole. It is the commitment to meet the challenges of the collaboration of the academia and key stakeholders to launch a significant attempt for developing a tailor-made process orientated approach on the basis of the current BIM technologies.

Although, research has been done based on the topics of BIM, yet limited research topics were conducted related to the topic of how to implementing BIM technologies when adopting robotic and automation technologies in the construction project. There are few questions need to be raised, such as, when implementing robotic and automation the operational methods and the working process will dramatically differ from the conventional way, so are the existing BIM technologies still adequate to handle the tasks? If not, is the proposed PIM concept able to tackle the challenges and how? This research forms the backbone for developing PIM applications in the future. However, due to the lack of available resources and complexity of the construction process, the PIM applications can only be conceptualised.

Consequently, the authors evaluate the current BIM and big data technologies through an extensive literature study to explore the potential constraints within each key project phases and exam how to transfer those constraints into opportunities for the construction sector and beyond. The proposed PIM concept offers a practical approach, which can be used as a guideline of how to integrate and distribute information and enhance decision-making procedures during the design, precedent and tendering, logistics, construction and facility management phases. As a result, the proposed PIM approach can yield a huge change in how the construction industry handles such large volumes of heterogeneous data as well as enhance information acquisition, integration, which provides real-time data sharing among all key stakeholders. In addition, it lays a foundation for developing a practical PIM application in the future.

\section{Building Information Modelling (BIM) and Process Information Modelling (PIM)}

\section{1. $B I M$}

Recently, BIM is one of the most promising developments in the Architecture, Engineering and Construction (AEC) industries. BIM became more influential within construction projects, which commonly used in the design, visualization, planning, facilities management and cost estimating purposes. Using modern modelling tools, such as Revit Architecture, ArchiCAD or Tekla Structures, the content produced by architects, designers and engineers have evolved from traditional 2D-drawings, sketches and written specifications to parametric, object-oriented 3D-models embedded with information to describe any building or facility in detail [6]. With BIM technology, an accurate 
virtual model of a building is digitally constructed [7]. When integrated efficiently, the computer-generated model contains precise geometry and relevant data needed to support the construction, fabrication and procurement activities involves in the project [3]. Although BIM applications claim the seamless integration of data from each project phase, in practice there is less evident how accurate and rapid the data has been passed on and how the data has been utilised. This may be resulted from the availability, compatibility and interoperability between raw data and the applications. These aspects determine if the data can be transferred, integrated and responded in real-time. On the other hand, BIM application is a knowledge-based and object-oriented approach that aims to digitally and visually represent the real world situations, in other words, it can be considered as the identical twin of the real world. This might sound unrivalled, however, when implementing construction robots or carry out a complex construction project, only understanding the real world condition is not enough. Know-how based, interactive, proactive and responsive extension of BIM is required [8].

\subsection{PIM}

The widespread use of digital technologies will lead huge amounts of data being generated throughout the construction process. Some of the data is well understood by the stakeholders, for instance, Computer-aided design (CAD) data, Excel data, 3D virtualizations. On the other hand, some of the other data may be less familiar to the stakeholders, and it hugely depends on their experience, background and professional field. The main objective of PIM is to make sure that everyone understands the data correctly; to predict what is going to happen in the future that based on the existing information variables. Specific actions need to be taken for analysing the risks and challenges might occur and recommending the options to the decision maker in real time [9].

PIM application is a process-oriented, case-focused approach that provides detailed information about a specific task. It then breaks down into smaller, manageable data and is distributed to the right stakeholder at the right time. The recipient can plan; react to the distributed data by following a guideline generated by PIM. The main feature of PIM is to optimize the entire construction process -rather than optimizing some parts and neglecting the others- by offering rapid, consistent data management and providing interactive, proactive, responsive data integration. In addition, it equips with cooperative and interoperable program applications that offer information, which can be understood by the stakeholders, rapidly accessible, predictive analytics as well as provides feasible guidance when issues occur [6].

In principle, PIM consists of five fundamental stages, which include Project break down, Data management, PIM Big Data (PBD) architecture construction, Implementation and PBD distribution. During the Project break down, each project stage is formulated as individual data cluster, which can be deployed, assessed, processed and transferred independently. For example, the initial data clusters shall include; design data, production data, procurement and tendering data, logistics data, rapid construction data and lifecycle management data. As a result, the data clusters are loosely coupled, they provided the database that can be categorized, classified and shared with the relevant party. The human data, physical data, project management data, facility data and cyber data will be analysed. The main goal of this stage is to differentiate and integrate the data based on the relevance of the information that evaluated by the key stakeholders. This action can potentially enhance interdepartmental, crossfunctional and cross-disciplinary data interaction; therefore, it adds value throughout the project.

During the next stage, PBD is further categorised into four main databases, which are physical database, BIM database, Internet of Building Things (IoBT) database and maintenance management database. The physical database contains the information that is gathered through paper-based hardcopy documents, as well as the information, which has not yet been transferred into digital data. The BIM database covers the range of information from basic data to highly sophisticated implementations, which include 3D, 4D, 5D and 6D BIM applications. The IoBT database comprises of a range of smart data collected throughout the construction phases, which include geolocation tracking, monitoring of equipment, inventory, procurement management, quality inspection, real-time measuring and control and remote operation. The maintenance management database covers the information accumulated over repairing, alteration, conversion, upgrading, scheduling and budgeting aspect of lifecycle management activities [9].

The aforementioned data is collected and stored in the PIM data Processing Unit (PPU). PPU is not only for data acquisition but also and most importantly it is a range of interoperable applications that actively processing data in real time and analysis huge amounts of data created from a variety of sources. The main strategy of PPU is to process, integrate, transfer, share and store the real-time data. Also, it enhances collaboration and supports the decision-making activities by distributing the most relevant information to the right key stakeholders at the right time. At the time of this writing, PIM is only developed as a conceptual model that demonstrates the overall concept, 
yet capable of offering basic instructions and data analysis that based on the scenario created by the Hong Kong CIC project.

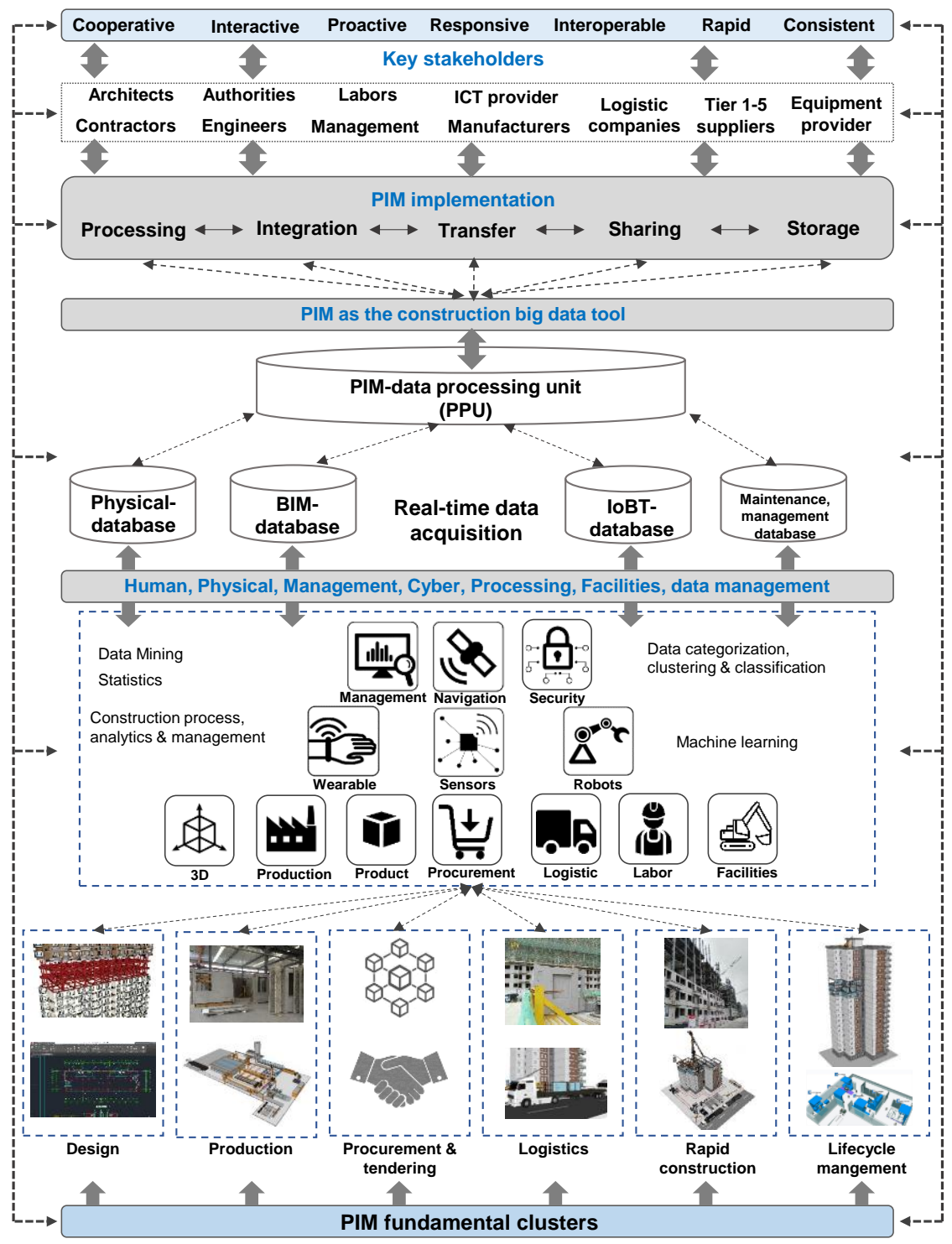

Fig. 1. Process Information Modelling concept.

\section{Literature Review}

The construction industry is dealing with significantly increasing data from varies disciplines throughout the construction process. As mentioned earlier, the PIM proposal can be seen as Big Data application. Utilisation of the applications can yield momentous benefits for an organization or individuals undertaking a variety of complex construction projects. In this section, the literature review and theoretical background are briefly introduced.

\subsection{Big data}

In general, Big Data is consists of two main activities, include Big Data Engineering (BDE) and Big Data Analytics (BDA). First, Big Data accumulates huge amount of data and then processing them by tools such as Apache Hadoop (AH), Apache Spark (AS). Secondly, Big Data storage is another crucial task, which manages whether or not the data is to be distributed or emerged. 
Apache Hadoop: The AH is an open-source implementation of MapReduce (MR) [10]. It is designed for managing very large node of the data base [11] on computer clusters that constructed from commodity servers. Nowadays, many web based brands are using Apache Hadoop platform, such as Yahoo! [12] and Facebook [13].

Apache Spark: The AS is one of the many open-source cluster computing platforms for processing large-scale data. It has gained increasing popularity recently due to the processing speed and user-friendly feature. It is a faulttolerant and optimised by Application-Programming-Interface (APIs) in interpreted high-level programming languages, such as Python, Java, R and Scala [14].

With the increasing amount of heterogeneous, autonomous parallel distributed sources and data, Big Data Analytics (BDA) has become crucial for many business disciplines. In general, BDA provides a new paradigm on how to handle, storage, manage and access to those huge datasets [15].

Starfish: Commonly, starfish is a self-tuning system used by data scientists, business analysists and IT operators to visualise, optimise as well as strategize the AH application. It builds on the AH application and adapting user's needs to achieve better performance automatically [16].

\subsection{SODATO}

SODATO stands for Social Data Analytics Tool that is developed to provide a generic method to gather, store, process, analyse and summarise big social data, which accumulates through the organisation's social media platforms. It provides a strategic tool that actively interacts with the big social data [17].

\subsection{Program language}

During the development of PIM concept, it was evident that there are various examples in computer software design, which have conceptual principles similar to those in PIM. A brief analysis is conducted to offer a reinterpretation of PIM concept by using software programing concepts [6].

Service-oriented architecture (SOA) can be described as a loosely coupled program architecture designed specifically to meet the needs of an organization [18]. By using communication protocols which provide services to another component and make connections between different software components over a network [19], the definition of a service can be viewed as a logical representation of a repeatable task. SOA is independent, self-contained, yet when combined it forms the functionality of a large software application. The unit architecture in PIM also shares similarities with the service architecture in SOA [20]. It is beneficial when linking an agent that influenced by the unit data or service, and the changes would influence the individual agent's capabilities or responsibilities [6].

The Microservice architecture is a programming concept inspired by SOA. Instead of traditional monolith software application, Microservices provides groups of independent program components that operated, deployed separately yet based on precise protocols and dedicated memories. The Microservice architecture has gain popularity in the recent years; it has potential to contribute to the development of PIM concept. However, there is limited researches have been conducted and emphasised the topic [21]. Therefore, further validation through application usecase is necessary [22].

Service discovery or service discovery protocols (SDP) is an emerging field in the area of ubiquitous computing [23]. They provide a mechanism, which allows automatic detection of service offered by any node in the network. In other words, service discovery is the action of finding a service provider for a requested service [24]. Service discovery can potentially operate as a search engine for the PIM architecture [6].

\subsection{System integration}

Due to the complexity of the construction projects, the multidisciplinary stakeholders and implementation of heterogeneous data through the use of different software and hardware are hard to manage. Data integration becomes very critical, which enables smooth operation and effective collaboration [25]. A number of challenges regarding to data integration within the construction, were identified by FIATECH [26], which can be summarized as below:

- There is a lack of transferability, interoperability between data, systems, programs and methodologies,

- The comprehensive universal management tool for different phases of the construction project is yet not available,

- Lifecycle management issues are often not emphasised. Operation, maintenance, dismantle and recycling are taken less consideration, 
- There is no common tool to manage health safety measures, and predictions of operation hazard are not available.

As a future construction IT system, PIM application will ensure construction information is available on demand and to be transferred to the desired stakeholders at the right time. All project partners, construction tools, equipment and machinery will be interconnected through integrated management systems. This will enhance planning, enable rapid response and optimize overall running of the project [27].

\section{PIM concept development for external wall painting task}

The Technical University of Munich (TUM) is commissioned by the Construction Industry Council Hong Kong (CIC) to research and develop construction robots and automation strategies that are tailor-made for the public housing construction project in Hong Kong. An extensive case study was conducted at Ngan Kwong Wan Road in Mui Wo, Hong Kong that erected by Hip Hing Engineering Co., Ltd. The project consists of one 14 stories and a 16 storey residential building. In this paper, the project team uses the 14 storey building as a case study to investigate how to implement PIM application to carry out external façade painting task by using a painting robot, which is developed during the CIC project.

First, the detailed breakdown of the working sequences and involved stakeholders and data for the exterior wall painting task are analysed. Whereas Figure 2 demonstrates the workflow for façade painting task carried out by a painting robot, Table 1 presents the corresponding data for each task description.

The main purpose of the proposed approach is to provide an automated process through accurate information flow from design phase to construction phase. Extension of the data stored in the BIM software is achieved by developing a property set. Pset_CIC_Painting is generated for schema IfcArchitectureDomain in the domain layer of the general IFC architecture. It has the properties of PaintingRobot, PaintingMaterial and MaxHeight. Data types for the criteria are set as IfcBoolean, IfcPropertyEnumeratedValue and IfcReal respectively. PaintingRobot finds out whether the façade painting is performed by a robot or not, MaxHeight refers to the maximum height of the external wall and PaintingMaterial includes the paint information.

CIC template file including the extended properties and building materials/composites for façade painting is created. The user should assign the each property using the IFC Manger menu. Pset_CIC_Painting is applicable to the project entity. Then, BIM file is exported as IFC format and transferred to an Excel file via IFC File Analyzer (IFA) [28]. In case of PaintingRobot being TRUE, the relevant data for the corresponding process can be extracted for the painting with automatic application.

Table 1. Robot-oriented painting task and related data.

\begin{tabular}{|c|c|}
\hline Task description & Data bank \\
\hline Installing the suspended working platform & $\begin{array}{l}\text { Product, logistic, planning, bidding, labour, equipment, health \& } \\
\text { safety, repair \& maintenance data }\end{array}$ \\
\hline $\begin{array}{l}\text { Delivering the painting robot and other } \\
\text { accessories }\end{array}$ & Robot, logistic, planning, bidding, navigation, distribution data \\
\hline Setting up \& calibrating the painting robot & Robot, planning, equipment, repair \& maintenance \\
\hline $\begin{array}{l}\text { Cleaning and preparing the external wall, skim } \\
\text { coating of the wall }\end{array}$ & $\begin{array}{l}\text { Robot, bidding, planning, equipment, repair \& maintenance, } \\
\text { labour }\end{array}$ \\
\hline Applying the first coat of paint & $\begin{array}{l}\text { Robot, paint supply, planning, equipment, repair \& maintenance, } \\
\text { labour }\end{array}$ \\
\hline Conducting quality inspection & Robot, planning, equipment, authority \\
\hline Applying the second coat of paint & $\begin{array}{l}\text { Robot, paint supply, planning, equipment, repair \& maintenance, } \\
\text { labour }\end{array}$ \\
\hline Conducting quality inspection & Robot, planning, equipment, authority \\
\hline Applying the final coat of paint & $\begin{array}{l}\text { Robot, paint supply, planning, equipment, repair \& maintenance, } \\
\text { labour }\end{array}$ \\
\hline Conducting quality inspection & Robot, planning, equipment, authority \\
\hline Finishing the areas where need to be painted again & Robot, paint, planning, equipment, repair \& maintenance, labour \\
\hline Dismantling the painting robot, hoisting device & Robot, planning, equipment, repair \& maintenance \\
\hline Dismantling the suspended working platform & $\begin{array}{l}\text { Product, logistic, planning, labour, equipment, health \& safety, } \\
\text { repair \& maintenance data }\end{array}$ \\
\hline
\end{tabular}




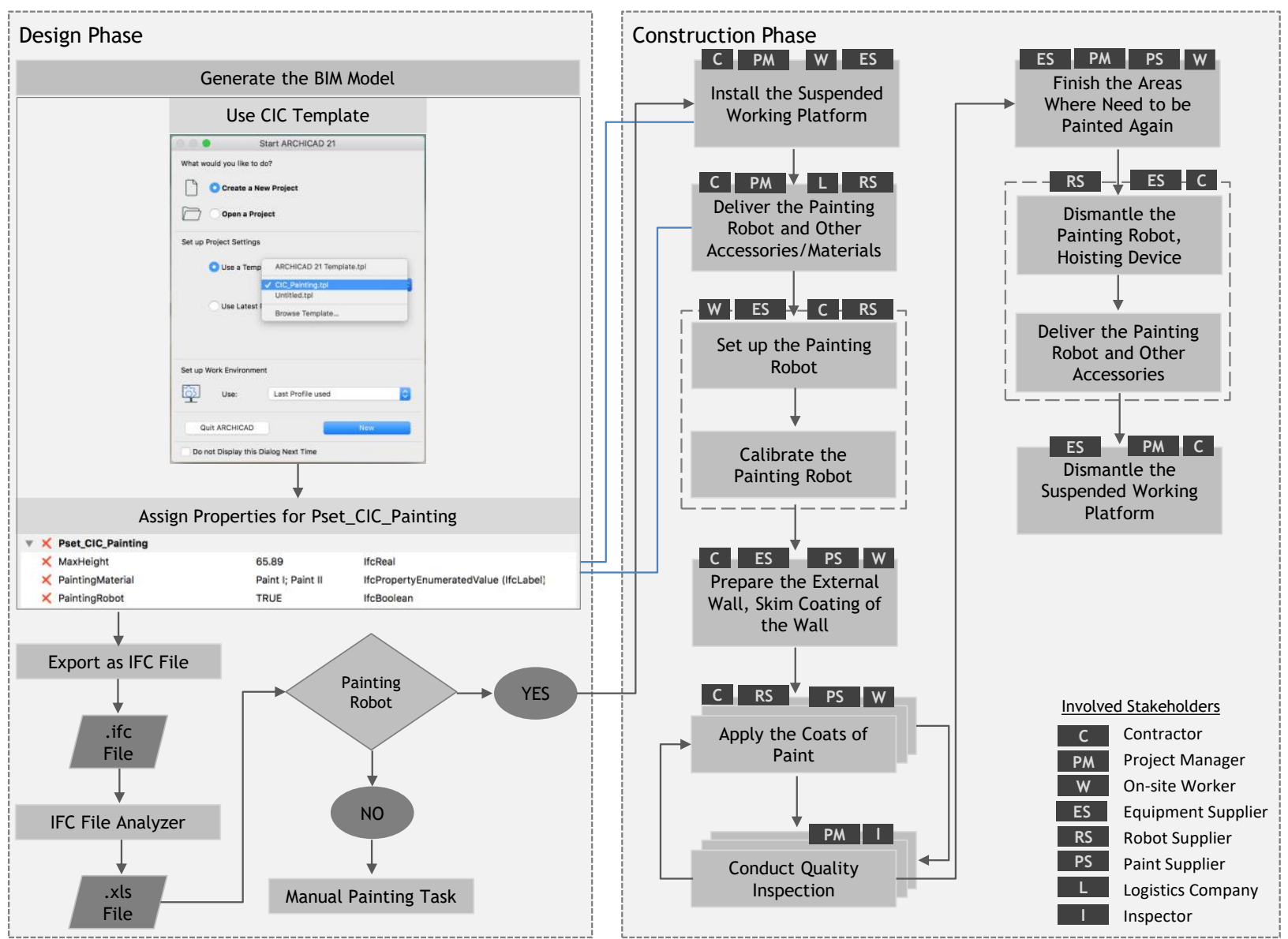

Fig. 2. PIM-based external wall painting task flowchart.

\section{Conclusion}

This paper provides an overview of the PIM concept and introduces a use case where how PIM applications would be operated while using the construction robotics on-site. The conventional BIM applications are designed to collect and distribute basic information about the construction project. However, they are not adequate in dealing with the situation if the construction robots are implemented. Since they have a limited function to understand the operational data generated by sensors and robots, there is a need of a more comprehensive approach. The concept of PIM enhances all aspects of the construction operations by not only collecting and distributing the data but also proceeding and analysing them for optimising the decision making during each task. The proposed approach provides systematic information flow and management for the construction phase of the project life cycle via incorporating the BIM data. This design and construction data integrated solution enables improved construction process management. In the current situation, BIM facilitates the design stage of a construction project and provides accurate project information, and continuation of the construction processes is separately handled based on the design data. Nevertheless, since most of the project delays and consequently cost overruns arise from the construction phase, more integrated and automated methodologies should be adopted. For instance, if the correct material/equipment information is gathered and transferred to the corresponding stakeholder as rapid as possible, the necessary actions can be made earlier than the actual construction to prevent any possible delays (e.g. in the façade painting case, checking the supplier of the paint automatically and in case of unavailability of that supplier, searching the possible ones via warning the related parties).

Moreover, the paper functions as an eye-opener to the construction industry through a demonstration on how to carry out BDE and BDA activities that associate with the vast amounts of heterogeneous data.

As mentioned earlier, at the time of this writing, it is limited to the conceptualisation of PIM. PIM is only developed as a conceptual idea that establishes the overall concept, yet will not bear on its ability of offering basic 
documentation tasks, provide instructions and data analysis by using existing Microsoft Excel tool. In addition, a comprehensive research project is needed to develop the concept further. It is important to use a real case study to develop the hardware and software environment, which required by PIM developers. Due to the given time and resources, the potential of the PIM application as a future Big Data application for the future construction industry, and privacy or data protection issues have not been detailed discussed.

\section{Acknowledgements}

The authors would like to sincerely thank the Construction Industry Council Hong Kong (CIC) and Hip Hing Engineering Co., Ltd for providing valuable information that supported this research.

\section{References}

[1] G. Higgin, N. Jessop, eds., Communications in the Building Industry: The Report of a Pilot Study, Routledge, 2013.

[2] C. S. Dossick, G. Neff, Organizational divisions in BIM-enabled commercial construction, Journal of Construction Engineering and Management. 136(4) (2009) 459-467.

[3] C. M. Eastman, P. Teicholz, R. Sacks, K. Liston, BIM Handbook: A Guide to Building Information Modeling for Owners, Managers, Architects, Engineers, Contractors and Fabricators, John Wiley and Sons, Hoboken NJ, USA, 2013.

[4] F.E. Jernigan, Big BIM, little bim: The Practical Approach to Building Information Modeling: Integrated Practice Done the Right Way!, 4site Press, 2008.

[5] E.L. Trist, K.W. Bamforth,. Some social and psychological consequences of the Longwall Method of coal-getting: An examination of the psychological situation and defences of a work group in relation to the social structure and technological content of the work system. Human Relations, 4(1) (1951), 3-38.

[6] W. Pan, K. Langosch, T. Bock, Development of the Process Information Modelling in the construction project: A case study of the ZEROPLUS project, in: Proceedings of $34^{\text {th }}$ International Symposium on Automation and Robotics in Construction, 2017.

[7] S. Azhar, A. Nadeem, J. Y. Mok, B. H. Leung, Building Information Modeling (BIM): A new paradigm for visual interactive modeling and simulation for construction projects, in: Proceeding of the First International Conference on Construction in Developing Countries, 2008, pp. 435-446.

[8] C. Harty, D. Throssell, H. Jeffrey, M. Stagg, Implementing building information modeling: a case study of the Barts and the London hospitals, in: Proceedings of the International Conference on Computing in Civil and Building Engineering. Nottingham (UK): ICCBE, 2010.

[9] P. Calie, Innovation Insight: The Impact of Emerging Technologies on the Construction Industry, DeltaHedron, 2017.

[10] J. Dean, S. Ghemawat, MapReduce: simplified data processing on large clusters, Communications of the ACM. 51(1) (2008) $107-113$.

[11] T. White, Hadoop: The Definitive Guide, O’Reilly Media, Inc., 2012.

[12] V. K. Vavilapalli, A. C. Murthy, C. Douglas, S. Agarwal, M. Konar, R. Evans, T. Graves, J. Lowe, H. Shah, S. Seth, B. Saha, Apache hadoop yarn: Yet another resource negotiator, in: Proceedings of the $4^{\text {th }}$ Annual Symposium on Cloud Computing. ACM, 2013.

[13] D. Borthakur, J. Gray, J. S. Sarma, K. Muthukkaruppan, N. Spiegelberg, H. Kuang, K. Ranganathan, D. Molkov, A. Menon, S. Rash, R. Schmidt, Apache Hadoop goes realtime at Facebook, in Proceedings of ACM SIGMOD International Conference on Management of data, 2011, pp. 1071-1080.

[14] M. Zaharia, R. S. Xin, P. Wendell, T. Das, M. Armbrust, A. Dave, X. Meng, J. Rosen, S. Venkataraman, M. J. Franklin, A. Ghodsi, Apache spark: a unified engine for big data processing, Communications of the ACM. 59(11) (2016) 56-65.

[15] J. A. Miller, C. Bowman, V. G. Harish, S. Quinn, Open source big data analytics frameworks written in scala, in: Proceedings of Big Data (BigData Congress), IEEE International Congress, 2016, pp. 389-393.

[16] H. Herodotou, H. Lim, G. Luo, N. Borisov, L. Dong, F. B. Cetin, S. Babu, Starfish: a self-tuning system for big data analytics, Cidr. 11(2011) (2011) 261-272.

[17] A. Hussain, R. Vatrapu, Social data analytics tool (sodato), in: Proceedings of International Conference on Design Science Research in Information Systems, 2014, pp. 368-372.

[18] A. Arsanjani, Service-oriented modeling and architecture, IBM Developer Works. 1 (2004) 15.

[19] T. Erl, Service-oriented Architecture: A Field Guide to Integrating XML and Web Services, Prentice Hall PTR, 2004.

[20] P. Patrick, A. Aletty, J. Kasi, C. Kapoor, T. Urhan, M. Mihic, Bea Systems, Inc. Service oriented architecture, U.S. Patent Application 11/133,022, 2005.

[21] H. S. Nwana, H.S., Software agents: An overview, The Knowledge Engineering Review. 11(3) (1996) 205-244.

[22] N. Dragoni, S. Giallorenzo, A. L. Lafuente, M. Mazzara, F. Montesi, R. Mustafin, L. Safina, Microservices: yesterday, today, and tomorrow, in: Present and Ulterior Software Engineering, Springer, Cham, 2017, pp. 195-216.

[23] G. G. Richard, M. Spencer, Service Discovery Protocols and Programming, McGraw-Hill Professional, 2001.

[24] S. E. Czerwinski, B. Y. Zhao, T. D. Hodes, A. D. Joseph, R. H. Katz, An architecture for a secure service discovery service, in: Proceedings of the $5^{\text {th }}$ Annual ACM/IEEE International Conference on Mobile Computing and Networking, 1999, pp. 24-35.

[25] W. Shen, Q. Hao, H. Mak, J. Neelamkavil, H. Xie, J. Dickinson, R. Thomas, A. Pardasani, H. Xue, Systems integration and collaboration in architecture, engineering, construction and facilities management: A review, Advanced Engineering Informatics. 24(2) (2010) $196-207$.

[26] FIATECH, http://www.fiatech.org/.

[27] Y. Rezgui, A. Zarli, Paving the way to the vision of digital construction: a strategic roadmap, Journal of Construction Engineering and Management. 132(7) (2006) 767-776.

[28] IFC File Analyzer (IFA), https://www.nist.gov/services-resources/software/ifc-file-analyzer. 